\title{
Modeling 3D Magma Dynamics Using a Discontin- uous Galerkin Method
}

\author{
Seshu Tirupathi ${ }^{1, *}$, Jan S. Hesthaven ${ }^{1,2}$, and Yan Liang ${ }^{3}$ \\ ${ }^{1}$ Division of Applied Mathematics, Brown University, Providence, RI 02912, USA. \\ 2 EPFL-SB-MATHICSE-MCSS, Ecole Polytechnique Fédérale de Lausanne (EPFL), \\ Lausanne, Switzerland \\ ${ }^{3}$ Department of Geological Sciences, Brown University, Providence, RI 02912, USA.
}

\begin{abstract}
Discontinuous Galerkin (DG) and matrix-free finite element methods with a novel projective pressure estimation are combined to enable the numerical modeling of magma dynamics in 2D and 3D using the library deal.II . The physical model is an advection-reaction type system consisting of two hyperbolic equations to evolve porosity and soluble mineral abundance and one elliptic equation to recover global pressure. A combination of a discontinuous Galerkin method for the advection equations and a finite element method for the elliptic equation provide a robust and efficient solution to the channel regime problems of the physical system in 3D. A projective and adaptively applied pressure estimation is employed to significantly reduce the computational wall time without impacting the overall physical reliability in the modeling of important features of melt and segregation, such as melt channel bifurcation in 2D and 3D time dependent simulations.
\end{abstract}

AMS subject classifications: 65Z05, 65M22, 65M60

Key words: magma dynamics, discontinuous galerkin method, , finite element method, matrixfree method.

\section{Introduction}

Generation and segregation of magma in the Earth and the interior of large planets has been a subject of intensive study in the Earth science community. Magma or melt can be generated by adiabatic decompressional melting during upwelling in the Earth's upper mantle and segregation of melt involves two-phase flow in which low viscosity melt percolates through a much more viscous solid matrix [6]. During its upward migration, melt generated in the deeper part of the upwelling mantle will interact both thermally

\footnotetext{
*Corresponding author. Email addresses: seshu_tirupathi@brown.edu (S. Tirupathi), jan.hesthaven@epfl.ch (J. Hesthaven), Yan_Liang@brown.edu (Y. Liang)
} 
and chemically with the overlying mantle. Our understanding of magma generation and segregation is constrained by physical evidence based upon geological field observations of ophiolites, where sections of the Earth's mantle have been uplifted and exposed at the surface. The key observation from these outcrops is the local depletion of a soluble mineral, orthopyroxene (opx). The elongated opx-depleted region, referred to as dunite in the geological literature, can be formed through a reactive infiltration instability, whereby highly porous regions form due to a positive feedback between melt percolation and dissolution of the soluble mineral opx in the solid. It has been suggested that high-permeability dunite channels act as conduits for focused flow, whereby melt may efficiently segregate from its source region while still maintaining its geochemical signature developed at depth. For further discussion we refer to $[12,13]$ and the references therein.

Previous numerical studies utilizing a low-order finite difference scheme was presented in [20], and demonstrated the localization of the melt flow into high porosity channels. However, this work does not explicitly account for the soluble mineral opx, whose presence is essential to the formation of dunite channels, nor does it consider the effects of upwelling. Further numerical studies incorporating upwelling of the mantle was presented in [19], although this work does not discuss the specific of numerical model used. In [17], a high-order accurate numerical model is presented based on the physical models presented in $[9,18]$, which include upwelling, a porosity-dependent bulk viscosity, and an additional equation to track the opx abundance. [17] also assumes a formulation of local chemical equilibrium with negligible diffusion in the melt. Linear stability analysis of this system predicts the emergence of compaction-dissolution waves [9]. In addition to the channeling instability, these features present a formidable challenge for numerical modeling. This challenge was previously addressed by developing a high-order numerical method that provides accurate resolution at a reasonable cost. A high-order discretization consisting of a tensor product of the Fourier collocation [17] or a high-order finite difference scheme [15] in the horizontal direction and discontinuous Galerkin methods in the vertical direction was used.

However, all previous work have employed a two-dimensional model and an expansion to the three dimensional problem would require a substantial effort. Rather than attempting this, we explore the use of existing libraries and, in particular, the software library called deal.II, offering multiple options such a high-order elements, support for adaptivity and the flexibility of expressing the problem in a dimension independent way [4]. The main objective of the present study is to develop an efficient and accurate numerical model that can be used to study the interaction between the melt and solid mantle during melt migration in both 2D and 3D. Following [17] we explicitly track the evolution of the soluble mineral (opx) to understand the formation of the observed opxdepletions and their spatial and temporal relation to localized melt flow in regions of high permeability. We evolve the governing system by solving the pressure equation using the current porosity values, and then evolve porosity and opx. While this method works perfectly to study the characteristics of melt migration, there are two significant 
bottlenecks in solving these problems in 3D. As the problem size increases, the memory footprint of the global pressure matrix significantly increases especially as the degree of the polynomial increases. The second issue is that the time required to solve the pressure equation significantly increases during the time evolution of porosity and opx. The first issue is addressed by using preconditioned matrix-free solution of finite element approximation for the pressure equation [14] and discontinuous Galerkin methods for the advection equations. Since all the operations in DG methods are local, there is no requirement to form a global matrix. The second issue is addressed by observing that the pressure only weakly affects the advection equations and an adaptive approach for solving the pressure equation is proposed to enable the solution of this global constraint only as needed. We shall also discuss the use of a projective pressure estimation to reduce computational cost of the iterative solver.

This paper is organized as follows. In Section 2 we outline the governing equations. In Section 3 we present the numerical discretization consisting of a discontinuous Galerkin scheme for the advection equation and finite element method for the pressure equation. This is followed by a description of projective pressure estimation methods to solve the pressure equation and derive an indicator to check the necessity of solving the pressure equation exactly. In Section 4 we show that results for 2D from transient numerical simulations match with what is predicted and observed in [17]. We then demonstrate melt channel bifurcation through transient numerical simulations in 3D under varying initial and inflow boundary conditions in porosity. Section 5 contains a brief summary and outlook.

\section{Physical model}

We consider a three-phase system with an interconnected melt or fluid phase and a solid that consists of an insoluble mineral, olivine (ol), and a soluble mineral, opx. We are interested in the spatial and temporal variations in the volume fraction of melt $\left(\phi_{f}\right)$, opx $\left(\phi_{o p x}\right)$, and the pressure difference between the melt and the solid matrix or effective pressure $p$ in this system. The governing equations of the model system have been previously discussed in $[2,20]$, and later extended in $[9,17,18]$ to incorporate upwelling, porosity-dependent bulk viscosity, and explicit tracking of opx. A reactive time scale was used in [9], whereas an upwelling time scale was used in [18]. Below we present the non-dimensionalized equations based on an upwelling time scale; we refer to $[17,18]$ for details of the non-dimensionalization and scaling.

The evolution of the melt fraction, $\phi_{f}$, and opx abundance, $\phi_{o p x}$, are described by the mass conservation equations,

$$
\frac{\partial \phi_{f}}{\partial t}=-\frac{\partial \phi_{f}}{\partial z}+\phi_{f} p+\delta \Gamma_{o p x}
$$




\begin{tabular}{|c|c|c|}
\hline Parameter & Symbol & Values \\
\hline \hline Normalized Solubility Gradient & $\delta$ & 0.01 \\
\hline Ratio of Melt to Solid Velocity & $R$ & 100 \\
\hline Initial Melt Fraction & $\phi_{f}^{0}$ & 0.02 \\
\hline Initial Opx Fraction & $\phi_{o p x}^{0}$ & 0.15 \\
\hline
\end{tabular}

Table 1: Input Parameters for the system

$$
\frac{\partial \phi_{o p x}}{\partial t}=-\frac{\partial \phi_{o p x}}{\partial z}-\frac{\phi_{f}^{0}}{\phi_{o p x}^{0}} \frac{\delta}{(1-\beta)} \Gamma_{o p x}
$$

Darcy's law yields the fluid velocity $\mathbf{v}$ relative to the uniform solid upwelling velocity as

$$
\phi_{f} \mathbf{v}=R^{-1} \phi \mathbf{n}_{z}-\phi_{f}^{3}\left(R^{-1} \nabla p-\mathbf{n}_{z}\right)
$$

where $\mathbf{n}_{z}$ is the vertical unit vector. Coupling Darcy's law to the momentum equation for solid matrix, one obtains the elliptic equation for the fluid-solid pressure difference $p$,

$$
-\nabla \cdot\left[\phi_{f}^{3} \nabla p\right]+\phi_{f} p=-R \frac{\partial \phi_{f}^{3}}{\partial z}
$$

Finally, under the assumption of local chemical equilibrium, the dissolution rate, $\Gamma_{o p x}$, is proportional to the Darcy flux, $\phi_{f} \mathbf{v}$, and is computed algebraically as

$$
\Gamma_{o p x}=\frac{\phi_{f}\left(\mathbf{v} \cdot \mathbf{n}_{z}\right)}{1-\delta z} \mathcal{I}_{o p x}
$$

where the indicator function $\mathcal{I}_{o p x}$ returns 1 if $\phi_{o p x}$ is positive and zero otherwise. In this way, dissolution occurs only if the soluble mineral opx is present.

\subsection{Parameter values, Initial and Boundary Conditions}

Following [18], we use a sustained perturbation in porosity at the inflow boundary of the domain. We take the boundary condition to be a Gaussian pulse added to unity,

$$
\phi_{f}(x ; y ; 0 ; t)=1+A_{1} \exp \left(-100\left(x-\frac{x_{\max }}{2}\right)^{2}\right)+A_{2} \exp \left(-100\left(y-\frac{y_{\max }}{2}\right)^{2}\right)
$$

where at least one of $A_{1}$ and $A_{2}$ is non zero in the examples discussed in Section 4 . For an initial condition we extend (2.6) across the entire vertical column, although results presented in this study are independent of the initial conditions except for the early time $(\mathrm{t}<\mathrm{R})$.

For the pressure equation we follow [9].

$$
p(x, y, 0, t)=-\frac{\delta(1+R)}{1+(n R)^{-1}}
$$


and zero Neumann condition at all the other boundaries. The input parameters used in this paper are listed in Table 1.

\section{Numerical discretization}

\subsection{Numerical Schemes}

To solve the system (2.1)-(2.7) we employ a discontinuous Galerkin (DG) scheme for the hyperbolic equations and continuous finite element method for the elliptic equation [14]. Time is discretized with an explicit, low storage, fourth-order Runge-Kutta (RK) scheme [7] which is simple to implement, requiring only function evaluations of the nonlinear right-hand sides to (2.1)-(2.2). Unlike previous numerical studies [20], our model assumes local chemical equilibrium and thus explicit time stepping is not restricted by a large reaction rate constant. The terms limiting the time integration are the upwelling and dissolution terms in (2.1) and are typically of comparable magnitude. Proceeding by a method-of-lines approach, the spatial operations in the hyperbolic equations (2.1)-(2.2) are discretized to produce a system of differential-algebraic equations that are a function of time only. Each inner RK stage is concluded by discretizing the elliptic equation (2.4) with the updated value of $\phi_{f}$. As a solver, we use a matrix-free implementation of BiCGStab iterative method for the elliptic equation [16].

\subsection{Full Numerical Method}

The domain $\Omega$ is triangulated into $\mathrm{K}$ non overlapping elements such that

$$
\Omega \simeq \Omega_{h}=\bigcup_{k=1}^{K} D^{k}
$$

The function space $V_{h}$ is defined as element wise discontinuous polynomials of degree $N$ such that

$$
V_{h}=\left\{v_{h} \in L^{2}(\Omega):\left.v_{h}\right|_{k} \in P^{N}(k): \forall k \in \Omega_{h}\right\} .
$$

We look for solutions $\phi_{f_{h}}, \phi_{o p x_{h}}$ and following the procedure in [11], the semi-discrete form of the porosity evolution (2.1) is

$$
\int_{D^{k}} v_{h} \partial_{t} \phi_{f_{h}}^{k} d \mathbf{x}-\int_{D^{k}} \phi_{f_{h}}^{k} \mathbf{n}_{z} \cdot \nabla v_{h} d \mathbf{x}=-\oint_{\partial D^{k}} v_{h}\left(\phi_{f_{h}}^{k}\right)^{*} \mathbf{n}_{e} \cdot \mathbf{n}_{z} d \mathbf{x}+\int_{D^{k}} v_{h} p \phi_{f_{h}}^{k} d \mathbf{x}+\int_{D^{k}} \delta v_{h} \Gamma_{o p x_{h}}^{k} d \mathbf{x}
$$

We choose an upwinding flux as

$$
\begin{aligned}
\phi_{f_{h}}^{k, *}\left(\phi_{f_{h}}^{k,-}, \phi_{f_{h}}^{k,+}\right) & =\phi_{f_{h}}^{k,-}, \mathbf{n}_{z} \cdot \mathbf{n}_{e} \geq 0 \\
& =\phi_{f_{h}}^{k,+}, \mathbf{n}_{z} \cdot \mathbf{n}_{e} \leq 0
\end{aligned}
$$




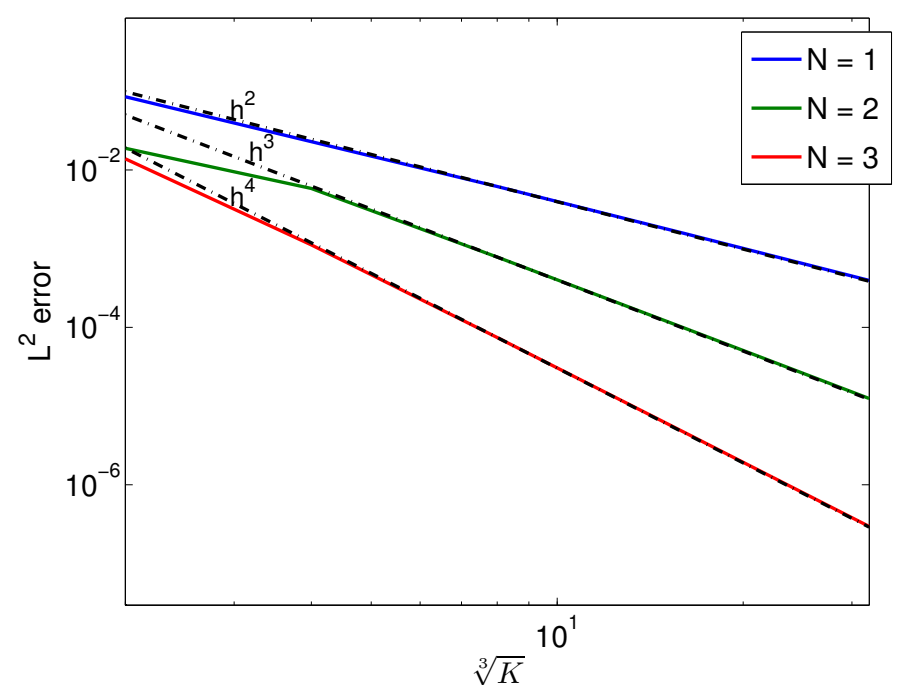

Figure 1: Convergence Study. Error Analysis with the manufactured solutions discussed in section 3.2 using Legendre Polynomials $P^{1}, P^{2}$ and $P^{3}$ respectively.

The opx evolution equation is discretized in a similar fashion

$$
\int_{D^{k}} v_{h} \partial_{t} \phi_{o p x_{h}}^{k} d \mathbf{x}-\int_{D^{k}} \phi_{o p x_{h}}^{k} \mathbf{n}_{z} \cdot \nabla v_{h} d \mathbf{x}=-\oint_{\partial D^{k}} v_{h}\left(\phi_{o p x_{h}}^{k}\right)^{*} \mathbf{n}_{e} \cdot \mathbf{n}_{z} d \mathbf{x}-\int_{D^{k}} \frac{\phi_{f}^{0}}{\phi_{o p x}^{0}} \frac{\delta}{(1-\beta)} v_{h} \Gamma_{o p x_{h}}^{k} d \mathbf{x}
$$

To verify the performance of the DG scheme we take the same parameter and domain setup as in the previous section, and assume an exact, steady solution as

$$
\begin{gathered}
\phi_{f_{e}}(x, y, z, t)=\exp (0.04 z)(1+0.1 \cos (2 \pi(x+y))) \\
\left.p_{e}(x, y, z, t)=p_{0}+0.1 \cos (2 \pi(x+y)) z \sin (0.75 \pi z)\right)
\end{gathered}
$$

Substituting these exact solutions into (2.1) and (2.4) yields a residual for each equation that is in turn added back to the equations, guaranteeing the exact solution. We integrate this system to steady state with very small time steps. From the general analysis of the DG method [11] we expect the error of the scheme to diminish as a polynomial function of the size of the element $h$. This is confirmed in Fig. 1 , where it is seen that the $L^{2}$ error decays as $h^{(N+1)}$ where $N$ is the degree of polynomial. Lastly, the elliptic equation is a modified Helmholtz equation which we choose to discretize using a standard finite element method, yielding the scheme

$$
\int_{\Omega} \phi_{f_{h}}^{3} \nabla p_{h} . \nabla v_{h} d \mathbf{x}+\int_{\Omega} \phi_{f_{h}} p_{h} v_{h} d \mathbf{x}=\int_{\Omega}-R \frac{\partial \phi_{f_{h}}^{3}}{\partial z} v_{h} d \mathbf{x}
$$




\begin{tabular}{|c|c|c|c|}
\hline \#Cells & \# dofs & \multicolumn{2}{|c|}{ Memory Consumption (MB) } \\
\hline & & Sparse Matrix & Matrix-free \\
\hline \hline $6,400(2 \mathrm{D})$ & 25,921 & 3.28 & 1.63 \\
\hline $40,000(2 \mathrm{D})$ & 160,801 & 20.50 & 11.10 \\
\hline $384,000(3 \mathrm{D})$ & $3,136,441$ & $1,581.07$ & 231.75 \\
\hline
\end{tabular}

Table 2: Comparison of memory requirements for the pressure equation

The pressure equation is solved using a BiCGStab matrix-free solver $[14,16]$ with the well known advantage of requiring less memory and eliminating the time for assembling. Table 2 gives a comparison between matrix-free methods and sparse matrix memory requirements.

\subsection{Projective Pressure Estimation}

While matrix free methods offer distinct advantages for solving large problems, the time to solve the pressure equation efficiently remains a bottleneck. Table 3 gives a distribution of wall clock times for the major tasks to be performed until opx channel is formed for a $2 \mathrm{D}$ case. As with most multi-phase flows, the advection equations weakly depend on pressure. Therefore, the computational wall time can be significantly reduced if the pressure is solved only when necessary. Such ideas have been pursued with solving the pressure equation once per fixed number of time steps [1] or utilizing the particular form of elliptic equations [8]. In this work we explore an adaptive indicator of the pressure equation, driven by a user defined tolerance $\delta^{*}$, and use this indicator along with a projective pressure estimation to estimate the pressure for cases where an exact solver is not required.

\begin{tabular}{|c|c|c|c|}
\hline Section & \# calls & Wall Time (s) & $\%$ Total \\
\hline \hline Assemble + Solve Hyperbolic Equations & 1,000 & $1.16 e+03$ & $21 \%$ \\
\hline Assemble Pressure RHS & 1,000 & 150 & $2.7 \%$ \\
\hline Output & 200 & 76 & $1.4 \%$ \\
\hline Assemble+Solve Pressure & 1,000 & $4.08 e+03$ & $74 \%$ \\
\hline
\end{tabular}

Table 3: Wall time distribution for major segments to evolve the system

Let $P^{n+1}$ be the pressure at the time step $n+1$ at which the pressure is needed and let $P^{n_{l}}$ be the last time step at which the pressure was calculated through a global solve. We denoted by $\underline{P}=\left[P^{n} P^{n-1} P^{n-2} \ldots . . P^{n-\left(n_{p p}-1\right)}\right]$ as the matrix of previous solutions and $n_{p p}$ is the number of previous solutions we have collected. We assume that these vectors are orthonormalized. Let $\mathbf{A}^{(\mathbf{n})}$ be the matrix that is generated by the numerical discretization of the elliptic operator and $f^{n}$, the right hand side at time step number $n$. 


\begin{tabular}{|c|c|}
\hline$n_{p p}$ & Average \# of iterations \\
\hline \hline 1 & 313 \\
\hline 2 & 112 \\
\hline 3 & 82 \\
\hline 4 & 73 \\
\hline
\end{tabular}

Table 4: Average number of iterations to solve the pressure equation with an initial guess using $n_{p p}$ previous solution vectors $\underline{P}$

If the tolerance of the iterative solver is given by $\epsilon$, then $P^{n}$ is the solution of the discretized PDE given as

$$
\left\|\mathbf{A}^{n} P^{n}-f^{n}\right\|<\epsilon
$$

Let

$$
\left\|A^{(n+1)} P^{n_{l}}-f^{n+1}\right\|=\delta
$$

and assume that $P^{n_{l}}=\underline{P} \alpha$, i.e. a simple linear combination of past pressure computations but with their linear combination recovered by satisfying the pressure problem at the current stage. If $P^{n+1}(x)$ does not vary rapidly or randomly with time, this linear combination should offer a good approximation, obtained at a limited cost. To gauge the accuracy of the projective pressure estimation, we need to find a criterion to decide if $P^{n+1}$ can be expressed through $\underline{P}=\left[P^{n_{l}} P^{n_{l-1}} P^{n_{l-2}} ..\right]$ or a full solution is required.

Generally we shall require

$$
\left\|P^{n+1}-P^{n_{l}}\right\|<C \delta^{*}
$$

where $C$ is a constant. Since we do not know $P^{n+1}$ we will estimate it in terms of known quantities.

$$
\begin{aligned}
\left\|P^{n+1}-P^{n_{l}}\right\| & =\left\|\mathbf{A}^{(n+1)^{-1}} f^{n+1}-P^{n_{l}}\right\| \\
& =\left\|\mathbf{A}^{(n+1)^{-1}} f^{n+1}-\mathbf{A}^{(n+1)^{-1}} A^{(n+1)} P^{n_{l}}\right\| \\
& \leq\left\|\mathbf{A}^{(n+1)^{-1}}\right\|\left\|A^{(n+1)} P^{n_{l}}-f^{n+1}\right\| \\
& \leq C \delta^{*}
\end{aligned}
$$

where $C=\left\|\mathbf{A}^{(n+1)^{-1}}\right\|$ and the user defined tolerance $\delta^{*}$ that can be checked in terms of known quantities is given by

$$
\left\|A^{(n+1)} P^{n_{l}}-f^{n+1}\right\|<\delta^{*}
$$

This criterion is cheap to check as it involves just one matrix-vector multiplication. Table 4 gives the average number of iterations for an $80 \times 80$ mesh for various choices of $n_{p p}$.

In the present study we take $\underline{P}=\left[P^{n} P^{n-1} P^{n-2}\right]$. Going further, since the porosity depends weakly on the pressure, we can further improve the computational speed by 


\begin{tabular}{|c|c|c|}
\hline$\delta^{*}$ & \# calls & Wall Time (s) \\
\hline \hline 0 & 1000 & $1.37 e+04$ \\
\hline 0.05 & 128 & $3.05 e+03$ \\
\hline 0.15 & 98 & $2.58 e+03$ \\
\hline 0.25 & 87 & $2.31 e+03$ \\
\hline
\end{tabular}

Table 5: Variation of wall time with user defined tolerance

solving the pressure equation exactly only when the residual $\delta$ is greater than the user defined tolerance $\delta^{*}$ and use the projected solution otherwise.

To summarize, details of the algorithm using projected pressure estimation to compute the pressure based on the user defined tolerance is given Algorithm 1.

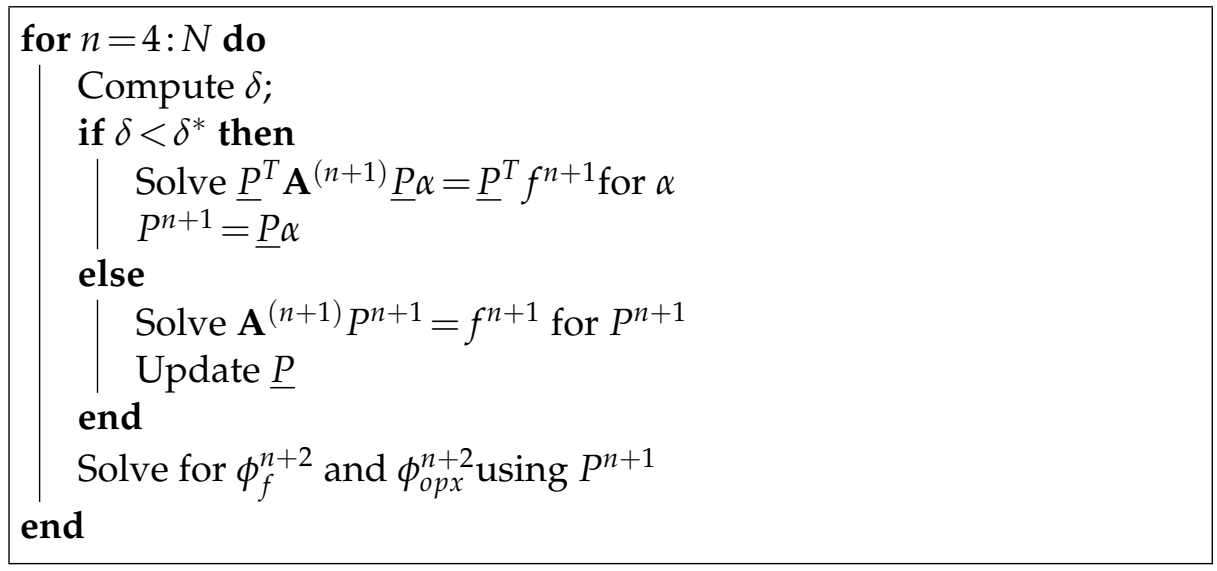

Algorithm 1: Projective Pressure Estimation for time steps $n \geq 4$

Table 5 gives the computational time to solve the given set of equations until opx becomes zero for a $40 \times 40$ mesh. We can see from the table that even though the number of calls to solve the pressure equation is less than $10 \%$ for $\delta^{*}=0.25$ as compared to solving the pressure equation at every time step, the gain in computational time is approximately six times. This is due to the the average number of iterations to solve the pressure equation increases as the projected solution slowly drifts further away from the actual solution. Fig. 2 gives a graphical comparison of the solutions at various times for various choices of $\delta^{*}$.

\section{Results from numerical simulations}

To demonstrate the accuracy, efficiency, and flexibility of the numerical scheme developed for the magma dynamics problem outlined in this study, we first present a set of examples of transient 2D simulations and compare our results with those reported in [18] 

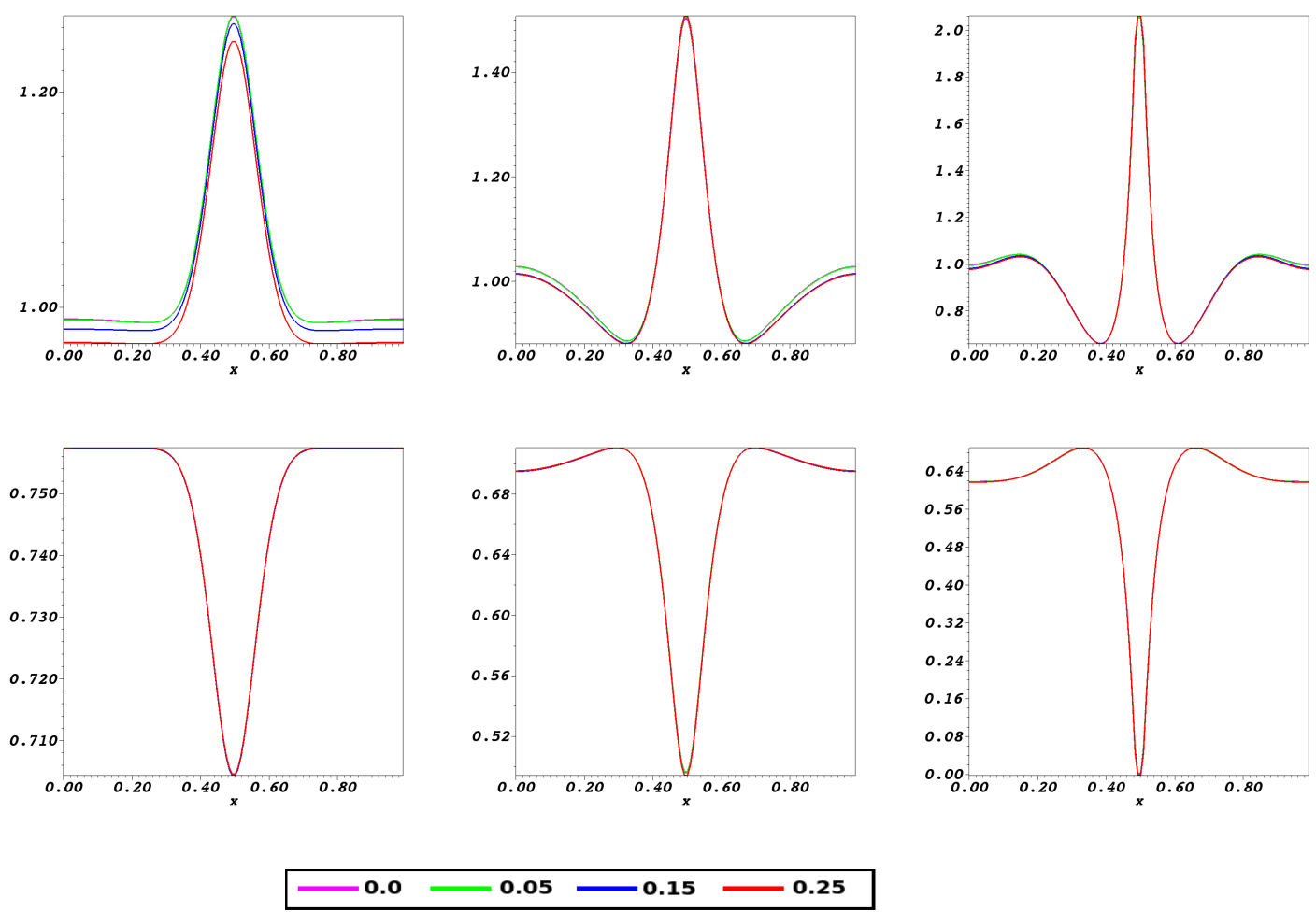

Figure 2: Time variation of solutions with sustained inflow perturbation for $\delta^{*}=$ $0,0.05,0.15,0.25$. Top: Fluid Fraction. Bottom: opx fraction. From left to right, $t=0.25$, $0.625,1.0$

for the same initial and boundary conditions. To further demonstrate the advantages of the numerical scheme, we then expand our simulations to 3D and briefly discuss their geological implications.

\subsection{Magma Dynamics in 2D}

The structure of steady-state high-porosity melt channels and their associated lithologies have been widely studied using high order accurate numerical methods in $2 \mathrm{D}[15,17,18]$. High order methods were employed to resolve the spatial variations of porosity and soluble mineral opx to the required numerical accuracy. Schiemenz and coworkers $[17,18]$ developed a mixed Fourier collocation - discontinuous Galerkin method for the magma dynamics problem described by Eqs. (2.1) - (2.7). In [18], extensive sets of 2D numerical simulations of high-porosity dunite channel formation in a domain of $1 \times 2$ dimensionless units (horizontal vs. vertical) were presented. The chosen width admits a minimum resolvable horizontal wave number of $\mathrm{k}=2$, placing the problem in a regime stable to porosity perturbation. Therefore we expect a steady state solution to exist. To form 

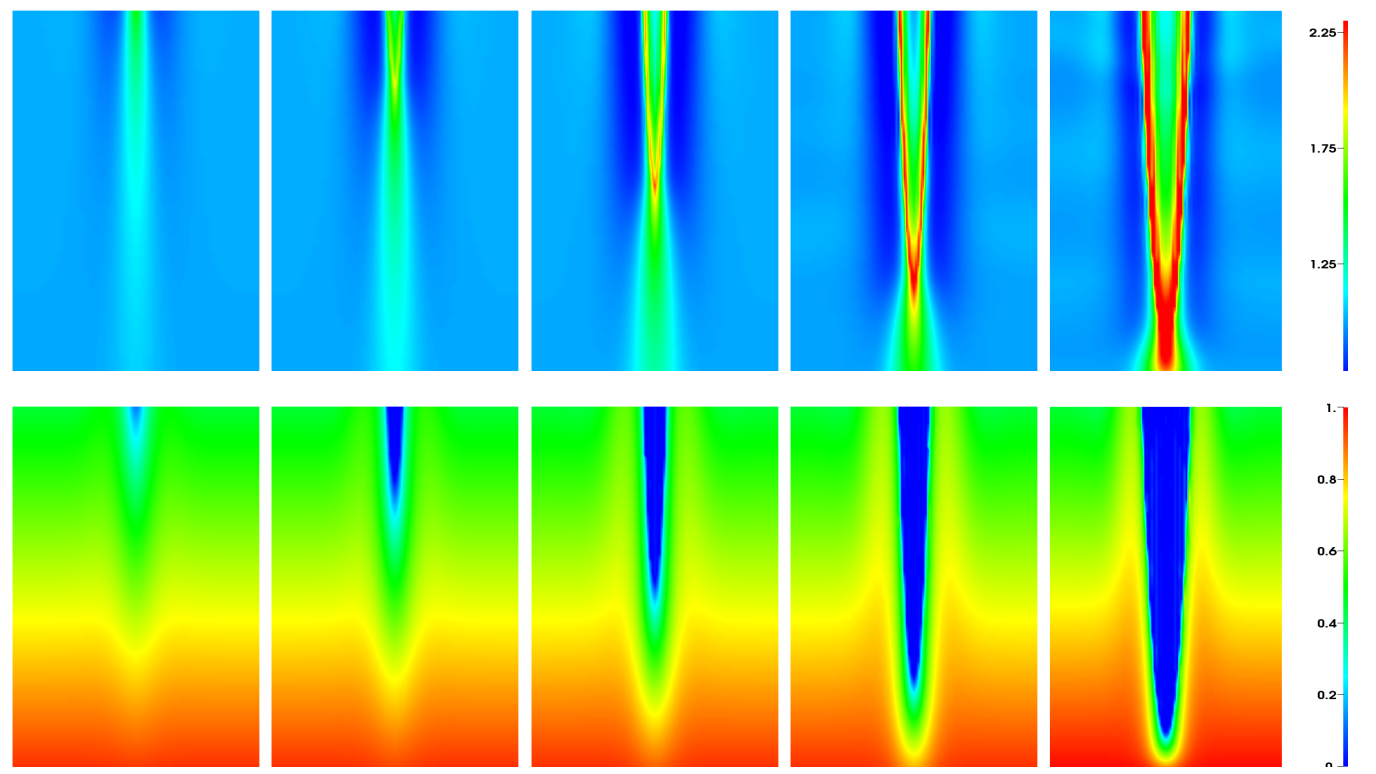

Figure 3: Steady State solutions with sustained inflow perturbation as given in case 2 . Top: Fluid Fraction. Bottom: opx fraction. From left to right, $\mathrm{A}=0.05,0.1,0.2,0.4,0.8$

high-porosity dunite channel in the stable regime, [18] imposed the following boundary condition at the inflow $(\mathrm{z}=0)$ :

$$
\phi_{f}(x, y, 0, t)=1+A \exp \left(-100\left(x-\frac{x_{\max }}{2}\right)^{2}\right)
$$

where $\mathrm{A}$ is the amplitude of the Gaussian-shaped porosity at $\mathrm{z}=0$. According to the 2D numerical simulations of [?], the imposed boundary condition (2.6) results in high porosity channels in the upper part of the simulation domain. Across the high porosity channel, large horizontal gradients in porosity, $\phi_{f}$, and opx fraction, $\phi_{o p x}$, are observed. In their numerical simulations, [18] uses $6^{\text {th }}$ order DG elements in the vertical dimension and a Fourier collocation scheme in the horizontal direction. This method consists of a uniformly high order method in the whole domain even though the prominent characteristics of $\phi_{f}$ and $\phi_{o p x}$ are in a small region near the channel boundary.

To compare with the $2 \mathrm{D}$ results of [18], we re-run a set of 5 simulations presented in Fig. 7 of [18] for the amplitude $A=\{0.05,0.1,0.2,0.4,0.8\}$ using the numerical scheme developed in this study. To better and more efficiently resolve large horizontal gradients, we use a highly non-uniform mesh. The advantage of such a variable mesh results is the reduction in the computational wall time. We can also consider a relatively low order method with this variable mesh to efficiently resolve the dynamics of the problem. In this case, spatial refinement gives the required numerical accuracy achieved through 
high order methods previously. Therefore, to negate the shortcomings in the numerical methods of $[15,17,18]$, we need numerical methods where there are no restrictions on element size and local approximation. For this reason, we use discontinuous Galerkin and finite element methods with a refined mesh near the channels and a coarse mesh otherwise. Fig. 3 displays steady-state distributions of the porosity (upper panels) and soluble mineral abundance ( $\phi_{o p x}$, lower panels) in the $2 \mathrm{D}$ domain for the five cases. These results agree very well with those reported in [18], including channel geometry (e.g., its width and depth), compacting boundary layers outside the high-porosity channels (deep blue regions in the upper panels), and melt channel bifurcation (two red branches within the dunite channels, upper panels). The larger perturbation or amplitude at inflow implies greater lateral porosity gradient leading to deeper and wider dunite channels. The total number of degrees of freedom in these cases are 15,750.

\subsection{Magma Dynamics in 3D}

All the simulations reported so far in literature are in simple 2D geometries. However, to compare with real field observations, we need to consider simulations in 3D. Extending the tensor product of Fourier collocation and discontinuous Galerkin method in 2D [?,?] may require a substantial effort. The numerical analysis library, deal.ii, makes it very easy to transform our code from $2 \mathrm{D}$ to $3 \mathrm{D}$ as the flux terms are treated in a dimension independent manner where lines and surfaces can be easily interchanged. A variable mesh becomes all the more important in 3D to control the overall cost. With a variable mesh, the number of degrees of freedom are substantially less than a uniform grid with a collocation method in the $\mathrm{x}$ and $\mathrm{y}$ direction. These factors result in a substantial improvement from previous numerical methods to solve magma dynamics problems in 3D. Fig. 4 shows the development of a tabular high-porosity dunite channel in 3D at three selected times and for the sustained inflow porosity

$$
\phi_{f}(x, y, 0, t)=1+0.15 \exp \left(-100\left(x-\frac{x_{\max }}{2}\right)^{2}\right)
$$

The computation domain is $1 \times 1 \times 2(x, y, z)$. Since the initial and boundary conditions are extended in the y direction (by one dimensionless unit), we observe the channel formation to be uniform along the y direction. Fig. 5 shows that the channel geometry, compacting boundary layer (deep blue regimes outside the tabular channels), and melt channel bifurcation are well resolved. Steady state is established for $t>2$. The degrees of freedom for this case are 704,000 with a non-uniform mesh as compared to a uniform mesh which would require 2,560,000 degrees of freedom to get the same channel features. In terms of physical features, the example shown in Fig. 5 is effectively 2D. To further demonstrate the advantages of our numerical method and importance of 3D simulations, we consider a simple 3D problem in a $1 \times 1 \times 2$ domain in which two sustained Gaussian perturbations in porosity at the inflow (bottom plane) are perpendicular to each other 


$$
\phi_{f}(x, y, 0, t)=1+0.1 \exp \left(-100\left(x-\frac{x_{\max }}{2}\right)^{2}\right)+0.1 \exp \left(-100\left(y-\frac{y_{\max }}{2}\right)^{2}\right)
$$

From 2D analysis, we know that the amplitude and shape of the perturbation at the boundary strongly affects the formation and characteristics of dunites in the stable regime. Fig. 5 shows that when the system reaches a pseudo steady state, there is a deeper cylindrical shaped channel at the center because of the higher amplitude of the Gaussian perturbation and 4 tabular channels in $\mathrm{x}$ and $\mathrm{y}$ because of the perturbations in each dimension respectively. Fig. 5 plots the snapshots of $\phi_{f}$ and $\phi_{o p x}$ at 3 different times prior to the solution reaching steady state. The spatial distribution of high-porosity melt channels and dunite channels in the Earth's interior is an important geological problem. Whether the 3D shape of dunite channels in the Earth's interior is tabular or cylindrical and if they are correlated with inflow perturbation are still not known. The numerical method developed in this study can be used to better characterize the geometry and spatial distribution of dunite channels in geologically more realistic settings, a topic that we are actively pursuing.

\section{Summary}

In this paper, a combined discontinuous Galerkin method and a finite element methods have been developed for the equations of magma dynamics describing the physical models presented in $[9,17,18]$. This model includes a porosity-dependent bulk viscosity term, a solid upwelling term and a hyperbolic equation to track the fraction of the dissolvable mineral opx. Our numerical implementation consists of a upwinding discontinuous Galerkin method for the advection equations and a classic finite element method for the elliptic equation. A projective pressure estimation was used to improve the computational time by making a better initial guess or by using an indicator to decide when to solve the pressure equation. This method is well-suited to study 3D channel regime characteristics which are predicted by linear analysis. Transient numerical simulations in 3D reveal new insight into the mantle heterogeneity. Future work involves extending these results to the wave regime and also study if projective pressure estimation methods can be applied to other weakly coupled hyperbolic-elliptic equations.

\section{Acknowledgments}

Seshu would like to thank Martin Kronbichler for valuable information on implementation of matrix-free methods and Marc Parmentier for helpful discussion and suggestions.

\section{References}

[1] E. Abreu, J. Douglas, F. Furtado, F. Pereira, Operating splitting for three-phase flow in heterogeneous porous media, Commun. Comput. Phys, 2009 


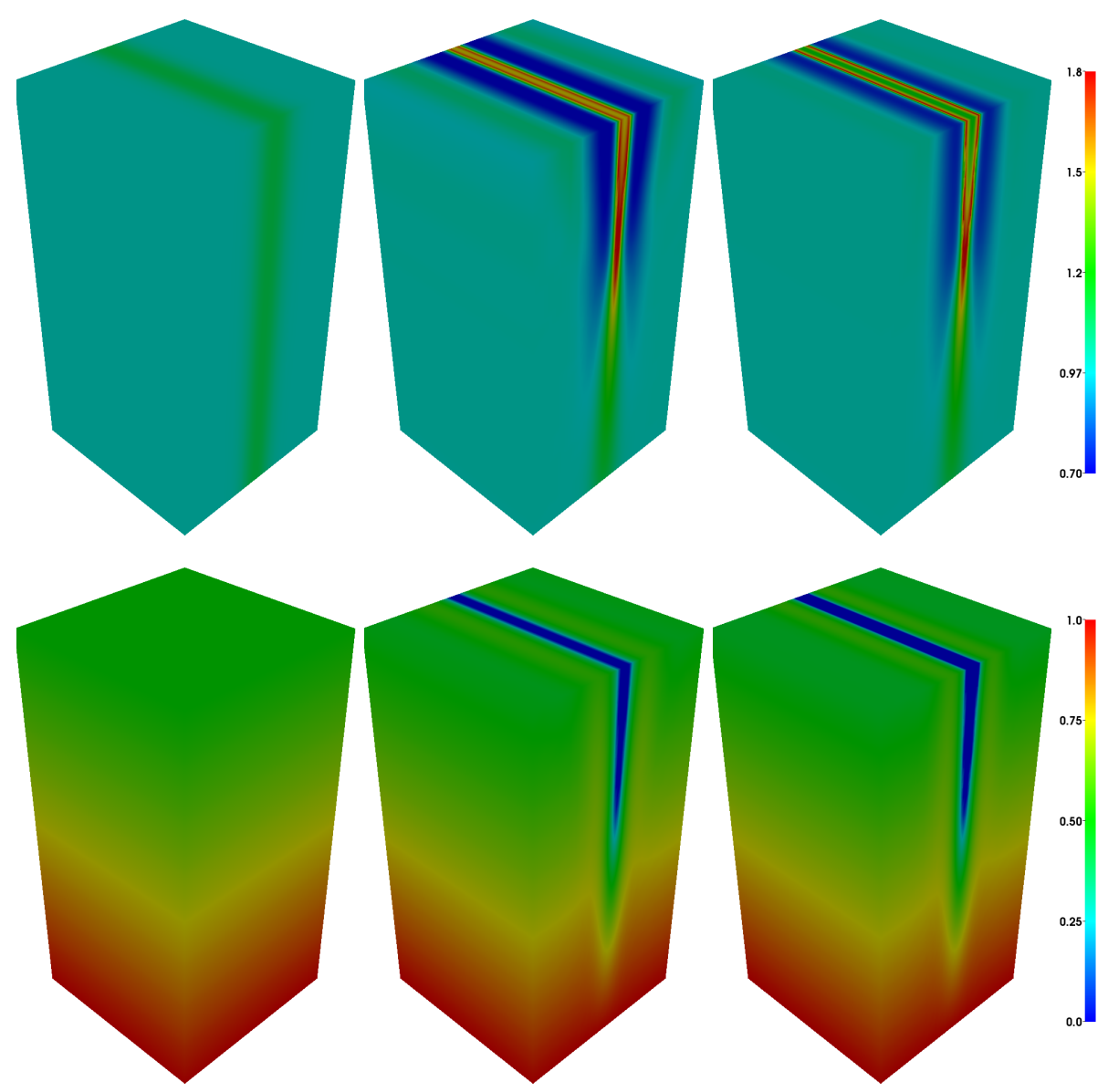

Figure 4: Time variation of solutions with sustained inflow perturbation that is uniform along y. Top: Fluid Fraction. Bottom: opx fraction. From left to right, $\mathrm{t}=0.0,1.2,2.4$

[2] E. Aharonov, J. Whitehead, P. B. Kelemen and M. Spiegelman, Channeling instability of upwelling melt in the mantle, J. Geophys. Res., 100 (1995), 433-450.

[3] D. N. Arnold, F. Brezzi, B. Cockburn and L. D. Marini, Unified analysis of discontinuous galerkin methods for elliptic problems, SIAMJ. Numer. Anal., 39 (2002), 1749-1779.

[4] W. Bangerth, R. Hartmann, and G. Kanschat, deal.II: A general-purpose object-oriented finite element library. ACM Transactions on Mathematical Software, 2007

[5] D. Bercovici, Y. Ricard and G. Schubert, A two-phase model for compaction and damage 1. General theory, J. Geophys. Res., 106 (2001), 8887- 8906.

[6] O. Buneman, Diagnosing oscillatory growth or decay, J. Comput. Phys., 29 (1978), 295-296.

[7] M. H. Carpenter and C. A. Kennedy, Fourth-order 2N-storage Runge-Kutta schemes, NASA TM 109112,NASA Langley Research Center, 1994.

[8] C. Chueh, N. Djilali, W. Bangerth. An h-adaptive operator splitting method for two-phase flow in 3D heterogeneous porous media. SIAM Journal on Scientific Computing, 2013

[9] M.A.Hesse, A. R. Schiemenz, Y. Liang and E.M. Parmentier, Compaction dissolution waves 

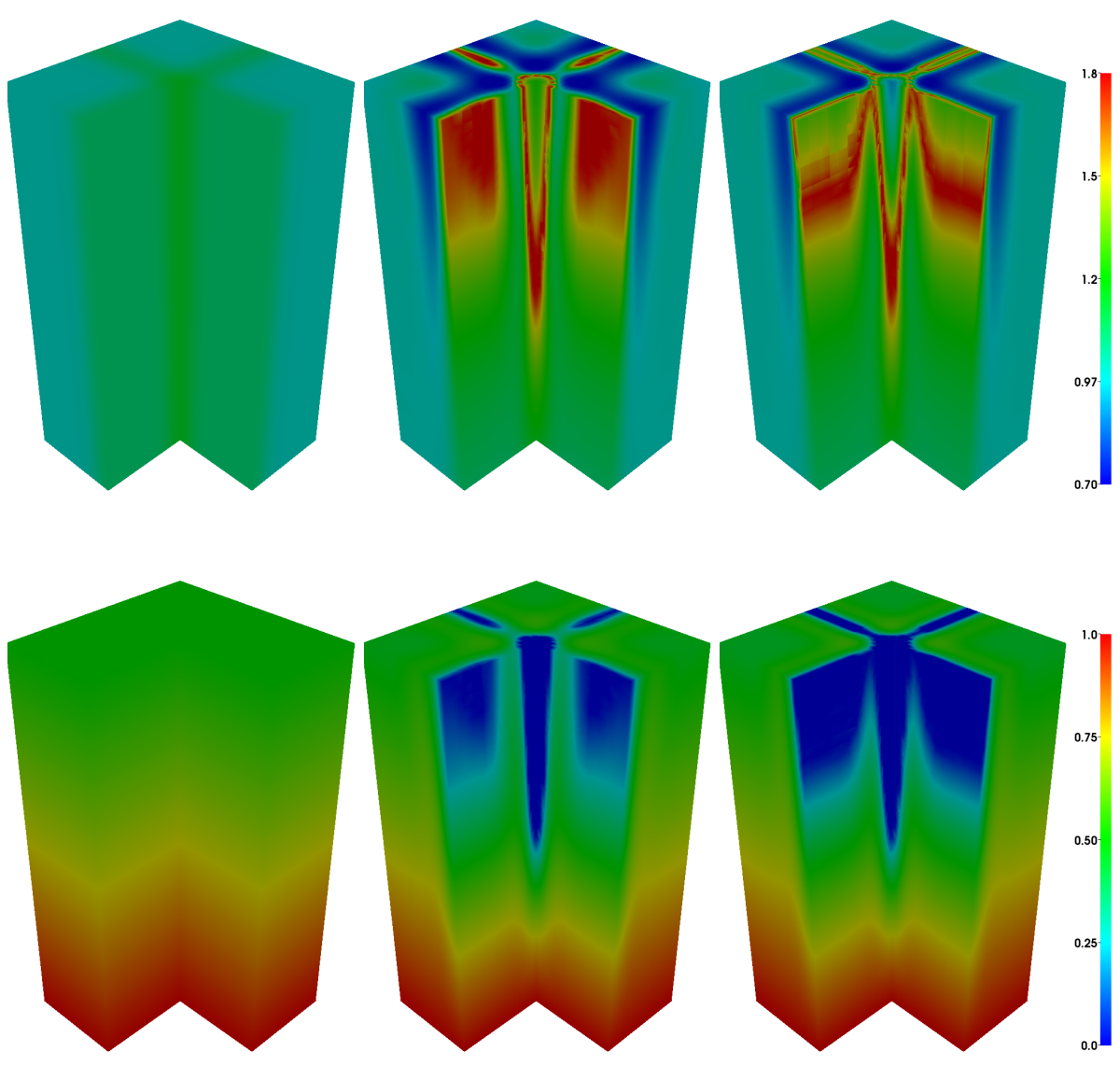

Figure 5: Time variation of solutions with sustained inflow perturbation in both $\mathrm{x}$ and $\mathrm{y}$ directions. Top: Fluid Fraction. Bottom: opx fraction. From left to right, $t=0.0,1.2,2.4$

in an upwelling mantle column, Geophys. J. Int., 2011 (submitted).

[10] J. S.Hesthaven, S. Gottlieb and D. Gottlieb, Spectral Methods for Time Dependent Problems, Cambridge University Press, 2006.

[11] J. S. Hesthaven and T. Warburton, Nodal Discontinuous Galerkin Methods: Algorithms, Analysis, and Applications, Springer, 2008.

[12] P.B.Kelemen, The origin of the land under the sea, Scientific American 300, no. 2, 52-57, February 2009.

[13] P. B. Kelemen, G. Hirth, N. Shimizu, M. Spiegelman and H. J. B. Dick, A review of melt migration processes in the adiabatically upwelling mantle beneath oceanic spreading ridges, Phil. Trans. R. Soc. Lond., 355 (1997), 282-318.

[14] M. Kronbichler, K. Kormann, A generic interface for parallel cell-based finite element operator application, Computers \& Fluids, 2012

[15] Y. Liang, A. Schiemenz, and M. Hesse, Waves, channels, and the preservation of chemical heterogeneities during melt migration in the mantle. 38, L20308, Geophys. Res. Lett., 2011. 
[16] Y. Saad, Iterative Methods for Sparse Linear Systems (2nd edition), SIAM Publishing, 2003.

[17] A.R. Schiemenz, M. A. Hesse, and J. S. Hesthaven, Modeling Magma Dynamics with a Mixed Fourier Collocation - Discontinuous Galerkin Method, Commun. Comput. Phys, 2011

[18] A. R. Schiemenz, Y. Liang and E. M. Parmentier, A high-order numerical study of reactive dissolution in an upwelling heterogeneous mantle: I. Channelization, channel lithology, and channel geometry, Geophys. J. Int., 2010

[19] M. Spiegelman and P. B. Kelemen, Extreme chemical variability as a consequence of channelized melt transport, Geochem. Geophys. Geosyst., 4(7) (2003), 1055.

[20] M. Spiegelman, P. B. Kelemen and E. Aharonov, Causes and consequences of flow organization during melt transport: The reaction infiltration instability in compactible media, J. Geo. Res., 106 (2001), 2061-2077. 